\title{
Pulp consistency determined by a combination of optical and acoustical measurement techniques
}

\author{
Matti Törmänen $^{1}$, Jan Niemi ${ }^{2}$, Torbjörn Löfqvist ${ }^{2}$ \\ and Risto Myllylä ${ }^{1}$ \\ ${ }^{1}$ Department of Electrical Engineering, 90014 University of Oulu, PL 4500, Finland \\ ${ }^{2}$ Department of Computer Science and Electrical Engineering, Luleå University of \\ Technology, SE-971 87 Luleå, Sweden \\ E-mail: matti.tormanen@oulu.fi
}

Received 28 October 2005, in final form 5 January 2006

Published 10 February 2006

Online at stacks.iop.org/MST/17/695

\begin{abstract}
In this study, methods based on ultrasonic attenuation and optical time-of-flight measurements are used simultaneously in determining both the fibres and fines mass fractions, respectively, of a cellulose pulp fibre suspension. The optical measurements are done by a laser radar and the acoustical measurements are based on ultrasonic attenuation measurements in a pulse-echo set-up. Two kinds of long-fibre fractions are studied, thermo-mechanical pulp and chemical softwood pulp. Fibre and fines mass fraction ranges are $0.25-1.0 \%$ and $0-0.75 \%$, respectively. The results show that the fibres are the predominant source for absorption and scattering of ultrasonic waves and are thus mainly contributing to the attenuation of ultrasound in the pulp. It is also found that the fines are the predominant source for optical scattering and fines are thus mainly contributing to the propagation delay of the light pulse in the laser radar set-up. By combining the ultrasonic attenuation and the optical time-of-flight measurements, it is shown that the mass fraction of fines and the mass fraction of fibres in a pulp sample could be determined, respectively.
\end{abstract}

Keywords: consistency, paper pulp, optical scattering, ultrasonic, acoustic

\section{Introduction}

In the paper industry, the requirements for higher paper quality, more effective use of raw material and higher paper machine speeds are demanding development of new measurement tools and methods. The situation is complicated by the increased use of recycled fibres, which leads to a situation where there is little or no information about the quality and type of fibres used in the process. This is vital information for the paper manufacturer in optimizing process control and maintaining a high product quality. To be able to achieve this, the paper manufacturer needs new and improved online process monitoring equipment to measure different process parameters.

The total mass fraction, or total consistency, of pulp is measured as the mass percentage of all dry material for a given pulp sample and is one of the most important control parameters for the paper maker. Today, there are a number of methods commercially available to measure total consistency online. Those methods are mainly based on attenuation and scattering of optical signals, attenuation and retardation of microwaves or changes in viscosity of the flowing pulp (Tornberg 1999). In all these methods, the calibration is recognized as the key to accurate consistency measurements (Tornberg 1999).

However, for online process measurements of the amounts of fibres and fines, respectively, there are no existing method available. In this paper, a new method for simultaneous fibre and fines consistency measurements based on the combination of laser radar and ultrasonic attenuation techniques is proposed. 


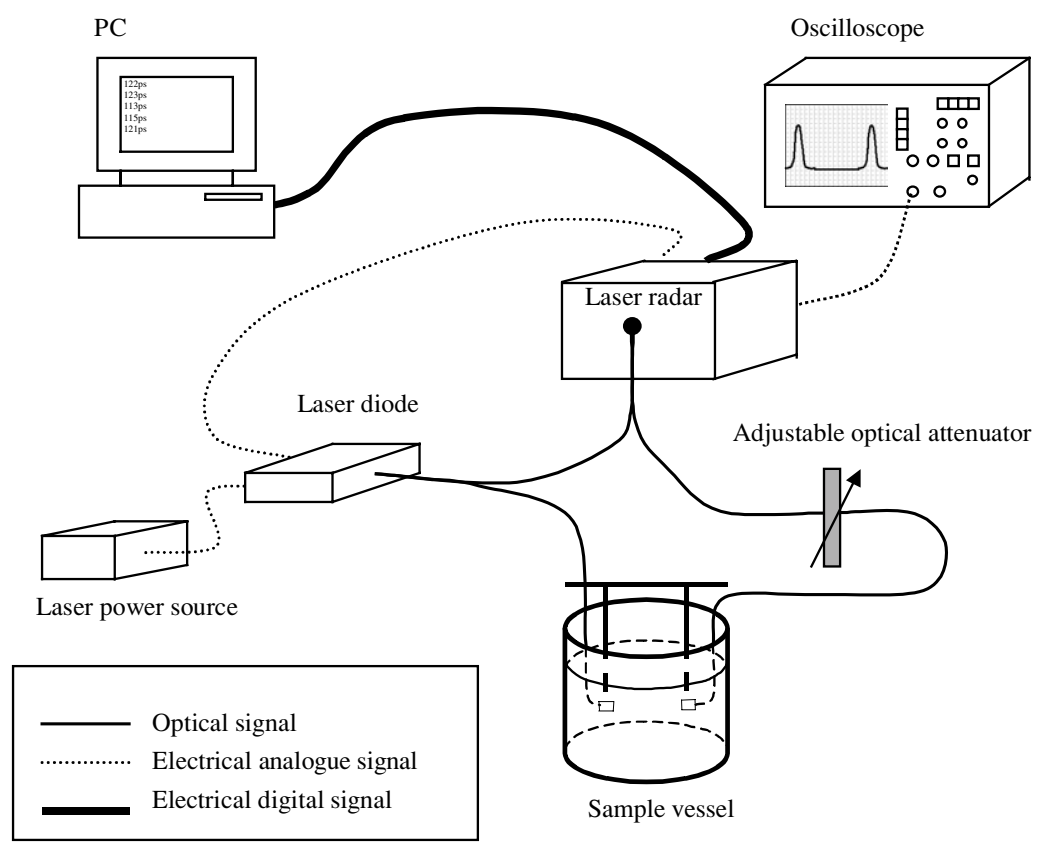

Figure 1. The optical measurement system set-up.

The proposed technique rests on the combination of two existing techniques, time-of-flight measurements of a light pulse and attenuation measurements of ultrasound. The timeof-flight is monitored by laser radar, which is based on measuring the propagation delay when a light pulse is sent through a sample of a certain thickness. The optical methods based on photon migration in a scattering medium are widely used in biomedical, atmosphere and seawater research areas (Yaroslavsky et al 2002). Theories describing the random walks of photons in a scattering medium are also widely known in the area of optical measurements (Yaroslavsky et al 2002). In the pulp and paper research area, the photon migration technique is used by Karppinen et al (1995), Carlsson et al (1995) and Saarela et al (2004).

The ultrasonic attenuation measurements method, used in this study, employs a pulse-echo technique where a short ultrasonic pulse is emitted by an ultrasonic transducer. The ultrasonic pulse is sent through a suspension sample, reflected at a reflector and subsequently received by the transducer. The pulse-echo technique is well known and is used, for instance, in medicine, quality control, process control and flow measurements. The theory for attenuation pulse-echo measurements for the set-up used in this study is described in McClements and Fairley (1992) and Löfqvist (1997).

The present study concerns the application of a combination of optical and acoustical measurement methods. The multi-sensor method proposed is designed to make measurements where fibre and fines consistencies are measured as separate variables.

\section{Materials and methods}

\subsection{Optical measurement system}

Light travels through a turbid medium in three different ways. It may propagate along a straight path, in which case we speak of ballistic photons, or along a slightly zigzagging path, referred to as snake light. Thirdly, light may be multiply scattered, in which case it is called diffuse light. In each way, light travels a different distance in the medium, and the time for propagation depends on the distance travelled and refractive index of the medium.

The propagation delay is formed from the changes in the photon trajectories caused by light scattering. The more are there scatterers in a medium, the longer the propagation delay will become. The photon propagation is commonly described theoretically by four optical parameters, namely scattering coefficient, absorption coefficient, anisotropy factor and refraction index. All of these are wavelength dependent.

The optical time-of-flight (TOF) measurement system consists basically of electrical circuitry, a laser radar, that performs a highly accurate time-difference measurement between two short optical pulses. The light source emits a light pulse that is split into two different paths having different optical pathways, see figure 1. The difference in optical pathway results in a time difference between the pulses when they arrive at the receiving circuitry in the laser radar.

The receiving circuitry sends a signal to a time-to-digital converter. This circuit calculates a time difference between the light pulses using a $100 \mathrm{MHz}$ clock and two integrators calculating the time in non-full start and stop pulses in the clock signal. The accuracy in measured time difference between each pair of light pulses is $1 \mathrm{ps}$.

The light source is a laser diode with a wavelength of $850 \mathrm{~nm}$ emitting an $8 \mathrm{~ns}$ long pulse at a repetition frequency of $10 \mathrm{kHz}$. The pulse is split and one part is guided to propagate through a pulp sample. On the receiving end an adjustable attenuator was mounted by which the optical power to the receiving circuitry was controlled. The control of optical power was necessary because of the limited dynamics of the receiver. The received optical power was displayed using an oscilloscope with $500 \mathrm{MHz}$ bandwidth. 


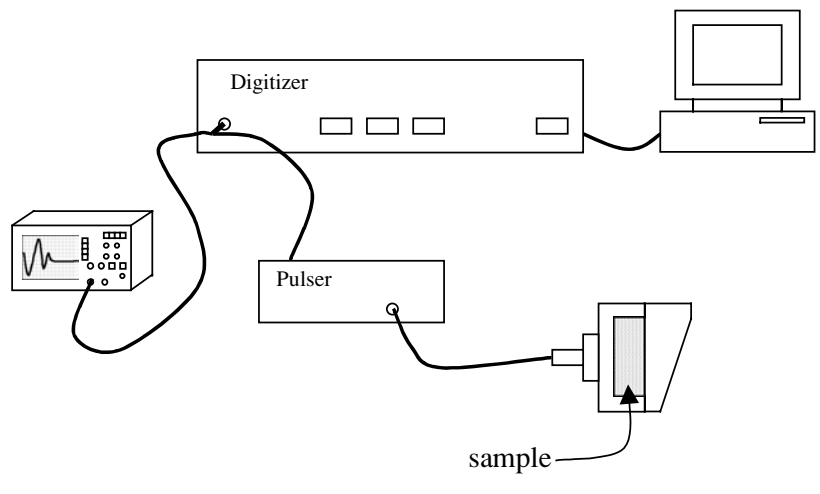

Figure 2. Ultrasonic measurement system set-up.

The system is equipped with a probe that can be immersed in the pulp sample. The probe consists of a transmitting and a receiving optical fibre. The receiving fibre is placed opposite to the transmitting fibre and along the same optical axis. The distance between the two fibres is adjustable. The optical fibres are not equipped with any focusing or collimating optics and the light from the transmitting fibre exits at the numerical aperture of 0.42 . The numerical aperture for the receiving fibre is 0.37 and its diameter is $1 \mathrm{~mm}$. This gives better dynamical behaviour compared to collimated light and the detecting fibre has a large diameter, which allows more scattered light to be detected.

During the measurement, the probe set-up is lowered into the pulp sample to a depth $35 \mathrm{~mm}$ from the surface of the sample. The free space around the probe is at least $35 \mathrm{~mm}$ in all directions. Note that the optical parameters of the pulp suspension are not investigated using the methods in this study.

\subsection{Ultrasonic system}

The attenuation of ultrasound derives mainly from the scattering and absorption of acoustic waves propagating through the suspension (Habeger 1982). They depend mainly on fibre size, mass fraction of fibres and the mechanical and thermal properties of the fibre material. A detailed treatment of scattering and absorption is quite complex and beyond the scope of this paper and it is in this study sufficient to lump the different acoustic attenuation mechanisms together in a frequency-dependent attenuation coefficient.

The ultrasonic measurement system consists of a customdesigned sample cell equipped with a piezoelectric transducer (figure 2). A detailed description of the cell, its operation and calibration procedure is found in Löfqvist (1997). For the present set-up, a transducer with $10 \mathrm{MHz}$ centre frequency, model V311, Panametrics, MA, USA, is used that spans a frequency range of 2-12 MHz. The transducer is mounted on a buffer rod made of cast plexiglass. The length of the buffer rod is $20.11 \mathrm{~mm}$ and its diameter is $30 \mathrm{~mm}$. The measurement cell was placed in a temperature-controlled room where the temperature is kept at $21 \pm 0.5^{\circ} \mathrm{C}$. The temperature in the room was monitored by a digital precision thermometer F250 MKII, manufactured by Automatic Systems Laboratories Ltd, UK. The digital thermometer was also monitoring the temperature of the pulp samples during the experiments. The thermometer was equipped with Pt-100 probes and accurate and traceable to $0.01{ }^{\circ} \mathrm{C}$.

A Panametrics 5052 pulser/receiver was used to excite the transducer as well as amplify the received signal. The received signal was digitized by a Sony/Tektronix RTD710 digitizer sampling at $200 \mathrm{MHz}$ with 10 bit resolution. The digitizer operated under software control from a computer and the digitized samples were, during each experiment, continuously stored in the computer for subsequent analysis. The received signal was monitored during the experiments using a Tektronix TDS720 oscilloscope.

The operating principle of the ultrasonic system is given by Löfqvist (1997) but reviewed briefly below for completeness. The transducer emits a short acoustic pulse. The pulse propagates through a buffer rod. At the interface between the buffer rod and the sample, a part of the pulse energy is reflected back to the transducer. The transducer that emitted the pulse records this first echo. The remaining part of the pulse propagates through the sample, reflects at a steel reflector and travels back through the sample and buffer rod and is subsequently recorded by the transducer as a second echo. Using these two echoes, the acoustic attenuation of the sample is determined. Since short acoustic pulses are used, the ultrasonic attenuation coefficient will be determined in a broad-banded frequency spectrum. In this study, the frequency range is found to be $1-10 \mathrm{MHz}$. The frequency range is set by the spectral content of the two received pulse echoes.

During the ultrasonic testing, the pulp sample under test is gently stirred in order to statistically reduce the uncertainties in ultrasonic attenuation due to flocculation. The sample is also stirred thoroughly prior to the test to eliminate the occurrence of air bubbles that might have been introduced while pouring the sample into the measurement cell. For each pulp sample, 100 ultrasonic pulses are recorded and averaged using an averaging procedure that reduces timing jitter (Löfqvist 1997).

\subsection{Samples}

The samples we used in this study were produced from thermomechanical pulp (TMP) delivered from UPM-Kymmene paper mill, Kajaani, Finland, and unbleached chemical Softwood pulp (SW) from Stora Enso paper mill, Oulu, Finland. First, the original TMP was fractionated by a Bauer-McNett fractionator according to standard SCAN-M6:69 (1969). The finest fraction, passing through the 200 mesh wire, was further filtered by 423 mesh wire sack. This fine fraction, consisting of particles between 30 and $74 \mu \mathrm{m}$, is used as the fines fraction in this study. The fines fraction consists of the particles that were collected in the mesh wire sack. In this kind of filtering, a high number of particles less than $30 \mu \mathrm{m}$ is lost; those would have a strong effect in optical scattering. As the long-fibre fraction we used the fraction rejected by 48 mesh wire from both TMP and SW pulps.

After the separation of fibres and fine particles to different classes, the fractions were mixed in previously determined proportions resulting in samples with a predefined range of fines and fibre contents. All three fractions were analysed in a Kajaani Fiberlab instrument, Metso Corporation, Finland, where fibre length distributions were obtained for each fraction. The resulting fibre length distributions for all three fractions are shown in figure 3. 

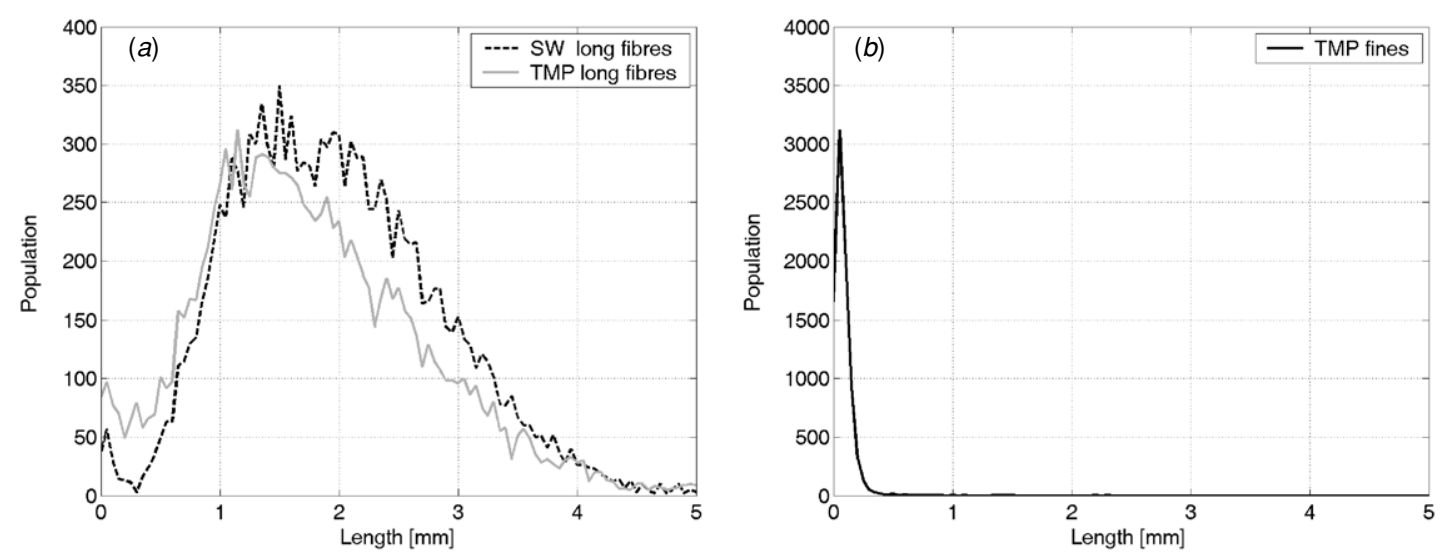

Figure 3. Fibre length distributions of filtered samples measured by the Kajaani Fiberlab. Fibre length distribution of long-fibre fractions is shown in $(a)$ and the length distribution of fines fraction is shown in $(b)$.

\section{Results}

Below, the fibre and fines mass fractions, or consistencies, are written as separate quantities. In all cases, the total consistency is the sum of fibre and fines consistencies. All the consistency values are expressed as mass percentages.

The notation of the individual samples is written in the following manner. First is the type of long fibres, secondly the fibre consistency and thirdly the fines consistency. For example 'TMP 0.50 .25 ' means the TMP sample consisting of $0.5 \%$ TMP long fibres and $0.25 \%$ fines. The notification 'TMP fib $0.25 \%$ ' or 'TMP fin $0.25 \%$ ' gives the constant amount of fibres or fines, respectively.

\subsection{Optical measurement results}

All the measured time-delay values are measured five times and the resulting delay value is calculated as the average of these five measurements. Between each measurement the pulp sample was mixed carefully. The standard deviations of each five measurements are expressed in error bars in figures.

As an example, some resulting measured time propagation delays are presented in figure 4. Generally, the delay values are a function of consistency and the distance between source and detector. The time-of-flight of a light pulse in water is subtracted from all measurements so the results are the additional time delay referred to pure water. The pulp samples in figure 4(a) consist only of the long-fibre fraction of TMP without any added fines. The four different curves show the pulse propagation delay at the consistency values: $0.25,0.50$, 0.75 and $1.00 \%$. The corresponding samples from long-fibre fraction of SW are shown in figure $4(b)$. In figure $4(c)$ time delays are shown where the long-fibre fraction of TMP is held constant and only fines are added to the samples.

If we inspect the time delay at a constant $25 \mathrm{~mm}$ separation between source and detector fibres, we obtain the curves shown in figure 5. In figure 5(a), the time delays are presented as a function of increased fibre consistency, the separate curves being pulp samples at constant fines consistency. The squares and triangles are TMP and SW samples without any fines, respectively. The crosses indicate the TMP samples having constant $0.25 \%$ fines consistency.

\subsection{Ultrasonic measurement results}

Representative examples of results from the ultrasonic attenuation measurements are shown in figure 6. In figures 6(a) and $(b)$, the ultrasonic attenuation for some long-fibre fractions of TMP and SW are shown for different consistencies. In figure $6(c)$, the consistency of the long-fibre fraction is held constant and fines are added. In the figures below, the ultrasonic attenuation is displayed as a function of the frequency of the ultrasound.

From the wide-banded ultrasonic measurements the attenuation value at $5 \mathrm{MHz}$ is extracted and presented in figure 7 where the results are presented as a function of fibre (figure 7(a)) and fines (figure 7(b)) consistencies. The estimate of the uncertainty of acoustic attenuation is $\pm 3.5 \mathrm{~m}^{-1}$ based on the 100 pulses at each measurement.

\section{Discussion}

\subsection{The optical time-of-flight method}

The mean fibre length in the TMP samples is shorter than in the SW samples, as seen from figure 1(a). Also, it is well known that the thermo-mechanical refining process roughens the fibre surface more which increases the specific surface area of the fibre. For these reasons, the TMP fibres cause higher light scattering and consequently larger propagation time delays compared to SW fibres. At $15 \mathrm{~mm}$ distance between source and detector, the recorded time-delay values are quite similar between each sample in figures $4(a)$ and $(b)$. The delay is some tens of picoseconds in pure water-which is used to normalize the results since only the delay difference from pure water is of interest.

When the distance between emitting and detecting fibres is increased the samples consisting of higher amount of TMP fibres begin to separate from the SW. This is due to the fact that ballistic photons are dominating in both samples TMP and SW at distance $15 \mathrm{~mm}$. At larger distances, the diffuse photons begin to dominate in the TMP sample.

The reason why SW fibres are not significantly increasing the delay in consistencies $0-1 \%$ is due to lower specific surface and higher absorption coefficient of SW fibres. The unbleached SW fibres are darker than TMP fibres. When 

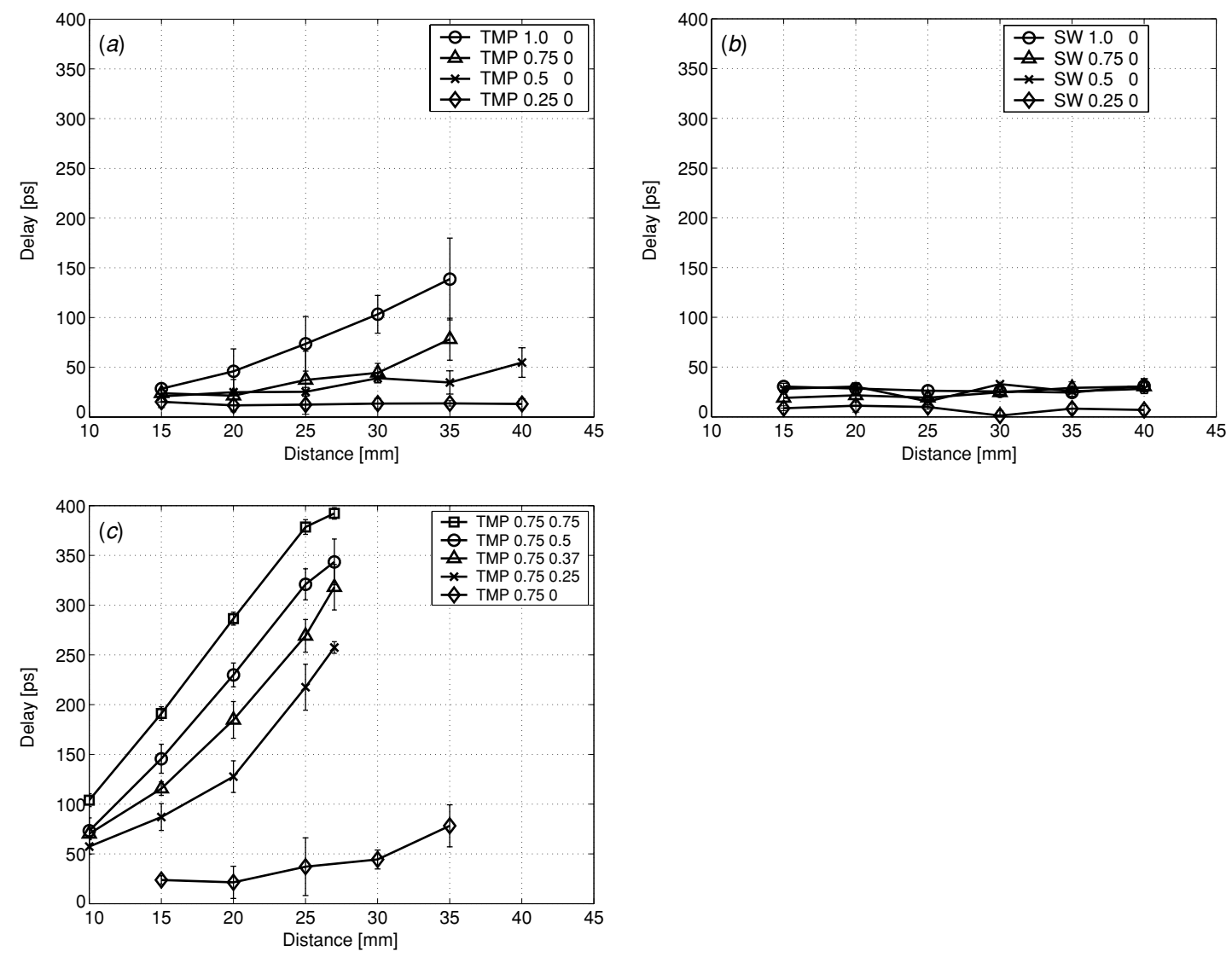

Figure 4. Optical propagation delays measured by laser radar at separate distances between source and detector. In (a) the TMP long-fibre fraction in four consistencies and in (b) SW long-fibre fraction in the same consistencies as in $(a)$ are shown. In $(c)$ TMP long-fibre consistency is constant and fines consistency is changed from 0 to $0.75 \%$.
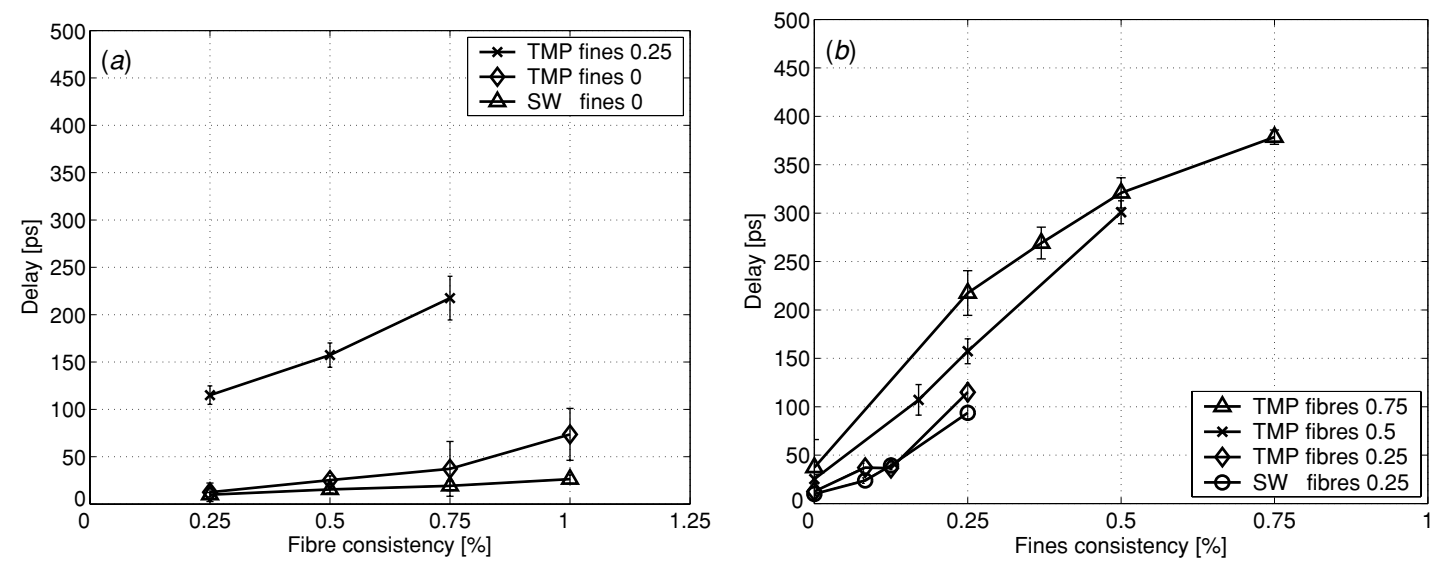

Figure 5. (a) Propagation time-delay values of TMP and SW long-fibre samples and TMP sample of $0.25 \%$ fines at $25 \mathrm{~mm}$ distances as a function of fibre consistency. In $(b)$ the fibre consistency is constant and fines is added.

photons interact with the dark surface, the probability of absorption instead of scattering is higher than in a bright surface as in TMP. The smooth surface of SW fibres is not promoting scattering and consequently most detected photons are moving along straight lines from source to detector. On the other hand, the higher optical absorption coefficient leads to a situation where multiply scattered photons travelling long distances are absorbed before they reach the detector.

Figure 4(c) shows the situation when the TMP longfibre consistency is held constant at $0.75 \%$ and fines is added stepwise from $0 \%$ to $0.75 \%$. Here, we clearly see the effect of the increased specific surface of sample on propagation delay if we compare the samples TMP_1_0 in figure 4(a) and sample TMP_0.75_ 0.25 in figure $4(c)$. We have two samples having the same dry weight but different particle size distributions and specific surfaces. The fines fraction is causing an additional delay at shorter distances than the same grammage of the long fibres. The adding of fines increases the delay.

In figure 5(a), delay values are shown when the distance between source and detector is constantly $25 \mathrm{~mm}$. The change 

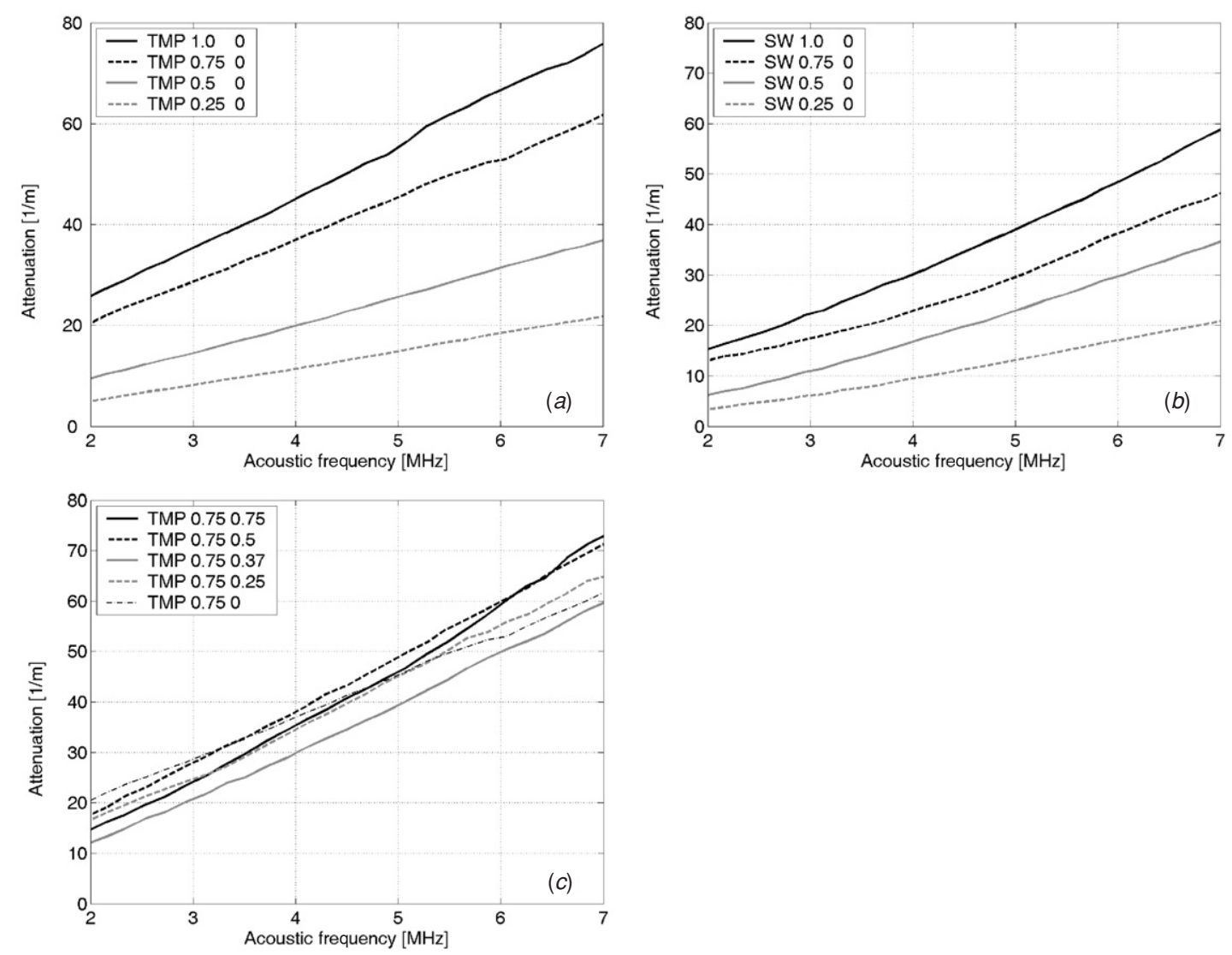

Figure 6. Acoustic attenuation measured as a function of frequency. In $(a)$ the TMP long-fibre fraction is shown for four consistencies and in $(b)$ the SW long-fibre fraction at the same consistencies as in $(a)$ is shown. In $(c)$ TMP long-fibre consistency is constant and fines consistency is changed from 0 to $0.75 \%$.
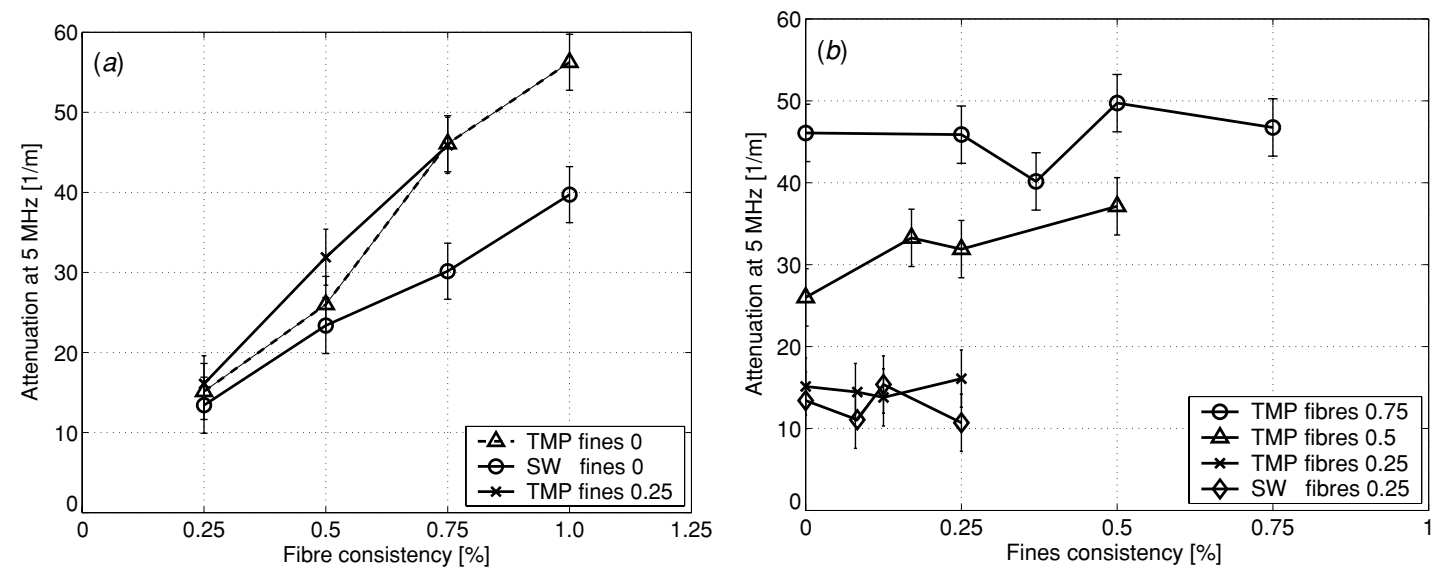

Figure 7. (a) Acoustic attenuation values of TMP and SW long-fibre samples and TMP sample of $0.25 \%$ fines at $5 \mathrm{MHz}$ frequency as a function of fibre consistency. In $(b)$ the fibre consistency is constant and fines are added.

of fibre consistency in the range of $0.25 \%-1.0 \%$ is not causing any significant time delay in SW. The effect of different fibre types is on view in pure fibre samples 'TMP fines 0 ' and 'SW fines 0 ' in figure 5(a). At 1\% consistency, the difference between delay values of pure TMP and pure SW is about $60 \mathrm{ps}$. The samples 'TMP fibres 0.75 ' and 'TMP fines 0.25 ' have about 140 ps longer delay than pure TMP at $1 \%$ consistency. Both these samples have the same dry weight.

The curves in figure $5(b)$ represent the constant fibre consistencies as a function of fines consistency. Here, we see that the TMP and SW samples in $0.25 \%$ fibre consistency follow the same trace. The increase of fibre consistency has less effect than the increase of fines and is shown in figure $5(a)$.

\subsection{The ultrasonic method}

In figures $6(a)$ and $(b)$, the acoustic attenuation caused by the long-fibre fractions without any fines is shown. From these figures, it is seen that there is a corresponding increase in 

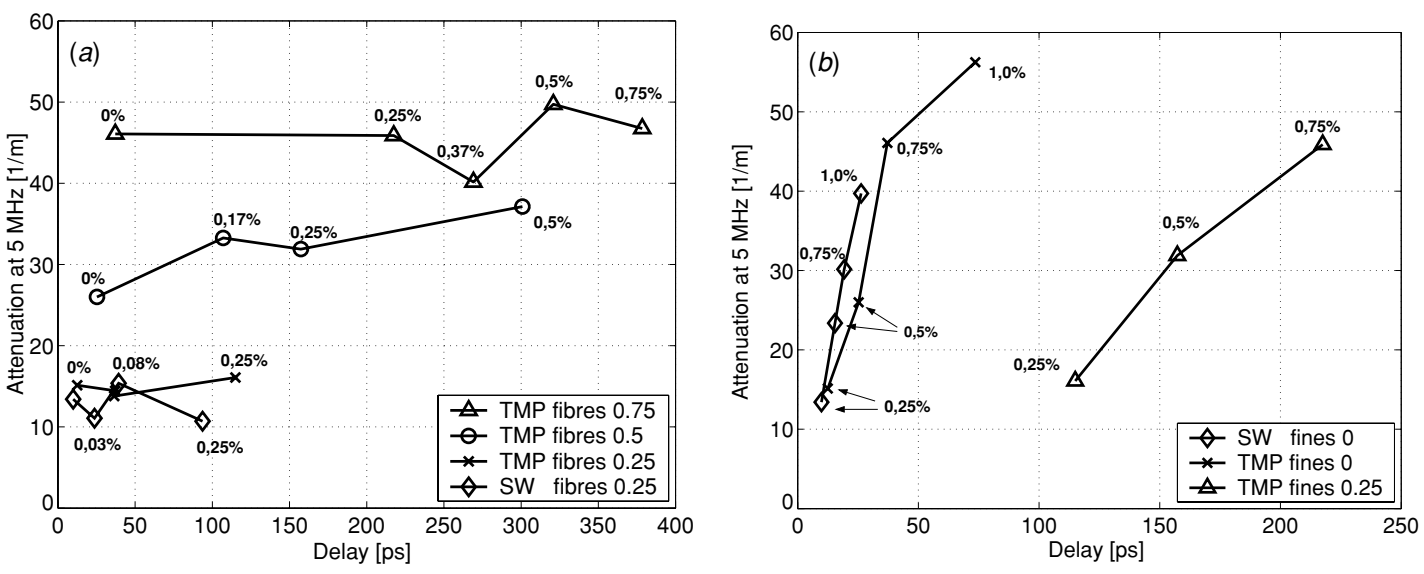

Figure 8. Acoustic attenuation as a function of optical propagation delay in different sample series. In (a) the amount of fibres is constant and the amount of fines is increasing from left to right. In $(b)$ the amount of fines is constant and the amount of fibres is increasing from left to right.

ultrasonic attenuation as the fibre consistency is increased. In figure $6(c)$, the ultrasonic attenuation is shown for the $0.75 \%$ TMP sample when the fines consistency is increased stepwise. In this figure, it is indicated that the increase in fines consistency is not changing the acoustic attenuation to any significant degree.

By using the measured attenuation at one frequency, in this study we have chosen $5 \mathrm{MHz}$, we can present attenuation as a function of the fibres consistency as shown in figure $7(a)$ or as a function of the fines consistency as shown in figure $7(b)$. From these figures, it is seen that the TMP fibres are attenuating the ultrasonic signal slightly more than the corresponding SW fibres at the same fibre consistency. This fine detail is small and believed to be of less significance to the results presented in this study. However, it could have a number of different explanations and thus calls for further investigations. The TMP sample containing $0.25 \%$ fines is not showing any significant increase in attenuation and stays close to the TMP sample with $0 \%$ fines.

In figure $7(b)$, the influence of fibre consistency is seen clearly. The TMP and SW samples having $0.25 \%$ fibres and varying amount of fines are found to be at close to constant attenuation coefficient level. Increasing fibre consistency causes the attenuation coefficient to increase to a higher value. It is also noted that increased amounts of fines have no significant effect on the attenuation coefficient. The same effect is seen at each of the different fibre consistencies.

When the optical and ultrasonic measurements are joined in the same plot, we obtain results as shown in figure 8(a) or $(b)$. From these figures, it is seen that using both the optical timeof-flight and ultrasonic attenuation methods simultaneously, it is possible to distinguish pulps having different amounts of fibres and fines, respectively.

The evident difference between fines consistencies of $0.25 \%$ and $0 \%$ is promoting the assumption that by measuring the ultrasonic attenuation it is possible to determine the consistency of long fibres from the total consistency of a pulp sample. With a simultaneous optical scattering delay measurement, it is possible to determine the amount of fines in paper pulp.
In this study, we have used only two pulp fractions: the fibres (rejected by 48 mesh wire) and fines (passed by 200 mesh wire). In real processes, the fibre size distribution is larger and continuous and there is also a larger span in mechanical and optical properties of the fibres. By using these methods, two variables are measured: the acoustic attenuation, mainly caused by long fibres, and optical propagation delay, mainly caused by the finest particles in pulp.

The optical and acoustical measurements are also complicated by the inhomogeneous nature of the pulp. For low consistencies, the fibres tend to sediment slowly and for higher consistencies they form flocks. Especially in samples consisting of long fibres, strong flocculation is noted. The flocks cause variations in the received signal since the flock represents a locally higher concentration of fibres blocking the optic or acoustic signal path. To minimize the influence from sedimentation and flocculation, the samples were stirred carefully and several readings were taken for each sample in order to obtain statistically significant results.

\section{Conclusions}

In this study, we found that by combining acoustic attenuation and optical propagation delay measurement methods it is possible to determine the amounts of fibres and fines, respectively, for a given pulp sample.

It is observed that both fibres and fines are causing optical propagation delay when added to a sample. But the increment of scattering caused by fines, is much larger than by fibres. It is also observed that the ultrasonic attenuation from fines is not as pronounced as the attenuation caused by fibres. These observations form the basis for the proposed technique to determine the consistencies of fibres and fines.

\section{References}

Carlsson J, Hellentin P, Malmqvist L, Persson W and Wahlström C-G 1995 Time-resolved studies of light propagation in paper Appl. Opt. 34 1528-35

Habeger C C 1982 The attenuation of ultrasound in dilute polymeric fiber suspensions J. Acoust. Soc. Am. 72 870-8 
Karppinen A, Kilpelä A, Karras M, Tornberg J and Myllylä R 1995 Papermaking furnish properties estimated by time-resolved spectroscopy J. Pulp Paper Sci. 21 151-4

Löfqvist T 1997 Ultrasonic wave attenuation and phase velocity in a paper-fibre suspension Proc. IEEE Ultrasonic Symp. (Toronto, Canada) pp 841-4

McClements D J and Fairley P 1992 Frequency scanning ultrasonic pulse echo reflectometer Ultrasonics 30 403-5

Saarela J, Törmänen M and Myllylä R 2004 Three methods for photon migration measurements in pulp Opto-Electron. Rev 12 193-7
SCAN-M6:69 1969 Fibre fractionation of mechanical pulp in the McNett apparatus SCAN-Test Methods (Stockholm: Scandinavian Pulp, Paper and Board Testing Committee)

Tornberg J 1999 Special measurements in pulp and paper processes Papermaking Science and Technology, Process Control vol 14: Process Control ed K Leiviskä (Helsinki: Fapet)

Yaroslavsky I V, Yaroslavsky A N, Rodriguez J and Battarbee H 2002 Propagation of pulses and photon density waves in turbid media Handbook of Optical Biomedical Diagnostics ed V Tuchin (Bellingham, WA: SPIE) 